\title{
Second differential of the entropy as a criterion for the stability in low-dimensional climate models
}

\author{
By T. PUJOL ${ }^{1 *}$ and J. E. LLEBOT ${ }^{2}$ \\ ${ }^{1}$ Universitat de Girona, Spain \\ ${ }^{2}$ Universitat Autònoma de Barcelona, Spain
}

(Received 22 April 1997; revised 15 June 1998)

\section{SUMMARY}

The second differential of the entropy is used for analysing the stability of a thermodynamic climatic model. A delay time for the heat flux is introduced whereby it becomes an independent variable. Two different expressions for the second differential of the entropy are used: one follows classical irreversible thermodynamics theory; the second is related to the introduction of response time and is due to the extended irreversible thermodynamics theory. The second differential of the classical entropy leads to unstable solutions for high values of delay times. The extended expression always implies stable states for an ice-free earth. When the ice-albedo feedback is included, a discontinuous distribution of stable states is found for high response times. Following the thermodynamic analysis of the model, the maximum rates of entropy production at the steady state are obtained. A latitudinally isothermal earth produces the extremum in global entropy production. The material contribution to entropy production (by which we mean the production of entropy by material transport of heat) is a maximum when the latitudinal distribution of temperatures becomes less homogeneous than present values.

KEYwORDs: Climatic stability Extremal entropy production Thermodynamic climatic models

\section{INTRODUCTION}

Climate modelling has largely been used for understanding the earth system, the sensitivity of the models being an important factor when studying the weight of parameters which determine the climate. Therefore, many authors have studied the stability of low-dimensional climatic models. North (1975a) and Ghil (1976), for instance, used the classical method of exponential disturbances which define the current state as stable, whereas e.g. Cahalan and North (1979) arrived at the same conclusion by using a Green's function. In all the models, however, the steady-state conditions for present, past or future climates are considered as stable. On the other hand, the analysis of the stability in classical thermodynamic systems is often studied by using the second differential of the entropy $\left(\delta^{2} s\right)$ as a Lyapunov's functional (Prigogine 1947). Hence, when the time $(t)$ evolution for $\delta^{2} s$ grows monotonically $\left(\mathrm{d}\left(\delta^{2} s\right) / \mathrm{d} t>0\right)$ the steady state reached for the system becomes stable.

The thermodynamic one-dimensional (1D) climatic model used in this paper follows North (1975b) where the diffusivity approach for the heat fluxes was applied; it is described in section 2. Usual 1D energy balance models (EBMs) assume a constant value for the conductivity parameter, but Nicolis and Nicolis (1980) realised that the climatic system does not satisfy a linear relationship between forces and fluxes. Here we have assumed that the heat flux is an independent variable following the criteria used in recently developed non-equilibrium thermodynamic theories. The evolution equation for the heat flux is a Maxwell-Cattaneo type of equation which can be seen as an extension of Fourier's law beyond stationary conditions (Jou et al. 1996). This simple modification permits us to obtain a completely different solution in low-dimensional climatic models (Pujol and Llebot 1999). Furthermore, Dedeurwaerdere et al. (1996) show that a more general expression for the evolution equation of the fluxes can be redefined through a Maxwell-Cattaneo equation by using an effective response time. This procedure has been very successful

\footnotetext{
* Corresponding author: Departament de Fisica, Universitat de Girona, Campus Montilivi 17071 Girona, Catalonia,
} Spain. 
for analysing non-equilibrium systems, and has been called extended irreversible thermodynamics theory (EIT; henceforward 'extended'), because a generalised entropy can be defined in terms of intensive parameters and dissipative fluxes (Jou et al. 1996).

In section 3 we present the results for the two expressions of the second differential of the entropy, the classical and the extended, comparing both results. For an ice-free earth and using high values of the response time, the results of the classical expression tend to a non-monotonic, growing, unstable state, as opposed to the well behaved extended case (always stable). However, the extended expression does not produce a monotonically increasing result for any value of the response time when the ice-albedo feedback is taken into account. In this case, the classical expression leads to unstable steady states when high response times are assumed. The extended expression produces some regions with high values of response time where the steady state becomes stable.

In addition to the thermodynamic study of the 1D EBM analysed, extremal rates of entropy production for present and ice-age conditions are obtained as functions of the albedo (section 4). The system is assumed to be stationary, and the maximum values are calculated through a variational principle (Nicolis and Nicolis 1980). The acceptance of an extremal behaviour for the climatic system would reduce the number of parametrizations assumed, simplifying the climatic evaluation in a given state. Thus Paltridge (1975) obtains a reasonable distribution of temperature, cloud cover and dissipative fluxes in a 1D box model (2D in Paltridge 1978) by using a maximum-dissipation hypothesis related to the material part of entropy production, by which we mean the production of entropy by material transport of heat (O'Brien and Stephens 1995). We show that the current rate of material entropy production is at least $20 \%$ lower than its maximum value. This deviation becomes larger if the unstable solution for present conditions is assumed. On the other hand, a latitudinally homogeneous temperature produces the maximum rate of global entropy production. In this case the difference from current values is less than $0.3 \%$, in agreement with Lesins (1990). Recently, O'Brien (1997) also shows the low sensitivity of the global entropy production to changes in dynamical processes.

\section{MODEL}

The thermodynamic model used is the 1D EBM developed by North (1975b) which is based in the first law of thermodynamics:

$$
C \frac{\partial T(t, x)}{\partial t}=-\nabla \cdot \mathbf{J}_{\mathrm{q}}(t, x)-\nabla \cdot \mathbf{R}(t, x)
$$

where $C$ is the heat capacity, and $\mathbf{J}_{\mathrm{q}}$ and $\mathbf{R}$ are the heat and radiation fluxes respectively. $T$ is the temperature which, like the fluxes, is a function of time and latitude. The coordinate $x$ is equal to the sine of the latitude. This horizontal 1D model is integrated along the vertical, and in (1) the dimensionless gradient operator follows:

$$
\begin{aligned}
& \nabla=\mathbf{a}_{x}\left(1-x^{2}\right)^{1 / 2} \frac{\partial}{\partial x} \\
& \nabla \cdot=\mathbf{a}_{x} \frac{\partial\left(1-x^{2}\right)^{1 / 2}}{\partial x}
\end{aligned}
$$

where $\mathbf{a}_{x}$ is a unit vector towards the pole. Therefore, the divergence of the radiation flux $\nabla \cdot \mathbf{R}$, expressed in $\mathrm{W} \mathrm{m}^{-2}$, is composed of the outgoing long-wave radiation $\nabla \cdot \mathbf{R}_{1+}(>0)$, 
incoming short-wave radiation $\nabla \cdot \mathbf{R}_{\mathrm{s}-}(<0)$ and outgoing short-wave radiation $\nabla \cdot \mathbf{R}_{\mathrm{s}+}$ $(>0)$ being:

$$
\begin{aligned}
& \nabla \cdot \mathbf{R}_{1+}=A+B \mathscr{F}(t, x), \\
& \nabla \cdot \mathbf{R}_{\mathrm{s}-}=-Q S(x), \\
& \nabla \cdot \mathbf{R}_{\mathrm{s}+}=+Q S(x)\{1-a(x ; T)\} .
\end{aligned}
$$

Equation (3a) is the linear parametrization of the long-wave radiation as a function of temperature $\mathscr{F}\left({ }^{\circ} \mathrm{C} ; T=\mathscr{F}+273 \mathrm{~K}\right)$, which can be accepted due to the range of temperatures considered and the effect of atmospheric long-wave absorption. With regard to the short-wave radiation, $Q$ is the solar constant divided by four, $S$ the insolation function and $a$ the co-albedo which takes a step-function form through the latitude of the ice-sheet edge.

The meridional heat flux in a diffusive EBM is taken to follow Fourier's law:

$$
\mathbf{J}_{\mathrm{q}}(t, x)=-\lambda_{\mathrm{q}} \nabla T(t, x),
$$

where $\lambda_{\mathrm{q}}$ is the planetary thermal conductivity.

In a system that is not in equilibrium (4) implies an infinite speed of propagation of thermal signals. Physically this is not a correct expression, and some attempts have been made to overcome this difficulty. One of the ways to obtain a finite speed of thermal pulses is to consider a Maxwell-Cattaneo type equation (Criado-Sancho and Llebot 1993):

$$
\tau_{\mathrm{q}} \frac{\partial \mathbf{J}_{\mathrm{q}}(t)}{\partial t}+\mathbf{J}_{\mathrm{q}}(t)=-\lambda_{\mathrm{q}} \nabla T(t)
$$

where $\tau_{\mathrm{q}}$ is the delay time of the heat flux. Equation (5) becomes the evolution equation of the heat flux within the framework of EIT, which has also been successfully applied in different fields (hydrodynamics, rheology, relativity etc.) enlarging the applicability of a macroscopic description in reference to classical irreversible thermodynamics theory (CIT; henceforth 'classical'; see e.g. Jou et al. 1996).

The expression for the climatic response time is obtained through a comparison with microscopic delay times. For example, the relaxation time for a monatomic gas is (Jou et al. 1996):

$$
\tau_{\mathrm{q}}=\frac{C_{\mathrm{C}} l^{2}}{\lambda_{\mathrm{q}}^{\prime}},
$$

where $l$ is the mean free path, $\lambda_{\mathrm{q}}^{\prime}$ the conductivity and $C_{\mathrm{C}}$ the heat capacity.

By including the horizontal thermal diffusivity of the system $k_{\mathrm{qh}},\left(k_{\mathrm{qh}}=\lambda_{\mathrm{q}}^{\prime} / C_{\mathrm{C}}\right)$, and defining the characteristic length $l$ as a function of the delay time $l=\tau_{\mathrm{q}} U$, then (6) becomes:

$$
\tau_{\mathrm{q}}=\frac{k_{\mathrm{qh}}}{U^{2}}
$$

where $U$ is the mean meridional velocity of the system analysed. By using current values of $k_{\mathrm{qh}}$ and $U$, the values of the atmospheric, mixed ocean layer and deep ocean layer response times are in accordance with Hasselmann (1979) $\left(\tau_{\mathrm{qa}} \sim 1\right.$ day, $\tau_{\mathrm{qm}} \sim 6$ months, $\tau_{\mathrm{qd}} \sim$ 1000 yrs, respectively). In this work we consider a fixed $\tau_{\mathrm{q}}$ along lines of latitude whose value can vary by changes in the meridional transport (i.e. changes in $U$ ).

On the other hand, the classical entropy is obtained from:

$$
T \mathrm{~d} s=C \mathrm{~d} T
$$


where $s=s\{T(t, x)\}$ which corresponds to the volume entropy integrated vertically (units $\mathrm{J} \mathrm{K}^{-1} \mathrm{~m}^{-2}$ ). Because $C$ is constant, integrating (8) gives:

$$
s(t, x)=C \ln T(t, x) .
$$

From (9) the second differential of the classical entropy yields:

$$
\delta^{2} s=-C\left(\frac{\delta T}{T}\right)^{2}
$$

where we have taken into account only first differentials and the product of them. As can be seen from (10), $\delta^{2} s$ is negative definite and, therefore, it can be chosen as a Lyapunov's functional (Jou et al. 1996).

The generalized entropy is a function of the intensive parameter $T$ and dissipative fluxes $\mathbf{J}_{q}$ and $\mathbf{R}$. In this case, however, we neglect the contribution of the radiative flux because a $1 D$ meridional vertically integrated model is used in the present study. Then, the response time of vertical fluxes (radiative fluxes) is assumed to be zero. Hence, the Gibbs equation of the generalized entropy, $s^{*}=s^{*}\left(T, \mathbf{J}_{q}\right)$ takes the form:

$$
T \mathrm{~d} s^{*}=C \mathrm{~d} T-\mathbf{A}_{\mathrm{qq}} \cdot \mathrm{d} \mathbf{J}_{\mathrm{q}},
$$

where to a first approximation $\mathbf{A}_{\mathrm{qq}}$ is a function only of $\mathbf{J}_{\mathrm{q}}$ :

$$
\mathbf{A}_{\mathrm{qq}}=\alpha_{\mathrm{qq}} \mathbf{J}_{\mathrm{q}} \text {. }
$$

The addition of more terms in (12) leads to a response time which is dependent on the heat flux. However, Dedeurwaerdere et al. (1996) show that the more general evolution equation for the heat flux can be qualitatively reduced to a Maxwell-Cattaneo expression (5) by using an effective response time.

The time derivative of the Gibbs equation in the form of (11), when (12) is used, is expressed as:

$$
\frac{\mathrm{d} s^{*}}{\mathrm{~d} t}=T^{-1} C \frac{\mathrm{d} T}{\mathrm{~d} t}-T^{-1} \alpha_{\mathrm{qq}} \mathbf{J}_{\mathrm{q}} \cdot \frac{\mathrm{d} \mathbf{J}_{\mathrm{q}}}{\mathrm{d} t} .
$$

Equation (13) has to be equal to the balance equation of entropy integrated along the vertical:

$$
\frac{\mathrm{d} s^{*}}{\mathrm{~d} t}=\mathscr{P}-\nabla \cdot \mathbf{H}-\nabla \cdot\left(\frac{\mathbf{J}_{\mathrm{q}}}{T}\right),
$$

where $\mathscr{P}$ is the rate of entropy production. $\mathbf{H}$ and $\mathbf{J}_{\mathrm{q}} / T$ are the radiation and material flux of entropy respectively. Substituting (1) in (13) and using (14), the rate of entropy production becomes:

$$
\mathscr{P}=\nabla \cdot \mathbf{H}-\frac{\nabla \cdot \mathbf{R}}{T}+\mathbf{J}_{\mathrm{q}} \cdot\left(\nabla \frac{1}{T}-\frac{\alpha_{\mathrm{qq}}}{T} \frac{\mathrm{d} \mathbf{J}_{\mathrm{q}}}{\mathrm{d} t}\right) .
$$

The coefficient $\alpha_{\mathrm{qq}}$ is obtained by assuming a bilinear form for the material entropy production*, whereby a similar expression to (5) is deduced:

$$
\alpha_{\mathrm{qq}}=\frac{\tau_{\mathrm{q}}}{\lambda_{\mathrm{q}} T}
$$

\footnotetext{
* There is still a great controversy with reference to the possible bilinearity of the radiation field. Two opposing views can be found in Callies and Herbert (1988) and Essex (1984).
} 
Then, the generalized Gibbs equation is:

$$
\mathrm{d} s^{*}=\frac{C}{T} \mathrm{~d} T-\frac{\tau_{\mathrm{q}}}{\lambda_{\mathrm{q}} T^{2}} \mathbf{J}_{\mathrm{q}} \cdot \mathrm{d} \mathbf{J}_{\mathrm{q}} .
$$

Taking into account that $T$ and $\mathbf{J}_{\mathrm{q}}$ are independent variables, the integration of (17) yields:

$$
s^{*}(t, x)=C \ln T(t, x)-\frac{\tau_{\mathrm{q}}}{2 \lambda_{\mathrm{q}} T^{2}(t, x)}\left\{\mathbf{J}_{\mathrm{q}}(t, x)\right\}^{2} .
$$

The first term on the right-hand side (r.h.s.) is the classical value of the entropy, (9). Equation (18) shows that the generalized entropy equals the classical entropy in equilibrium. However, in the steady state the generalized entropy presents a lower value than the classical expression. The difference between classical $(s)$ and extended (generalized) entropy $\left(s^{*}\right)$ is the second term on the r.h.s. in (18) if the heat flux is the unique dissipative flux in the system.

On the other hand, the rates of entropy production in stationary conditions take the same value in both extended and classical cases, but the time evolution outside the steady state does not. From (18), the second differential of the generalized entropy becomes:

$$
\delta^{2} s^{*}=-C\left(\frac{\delta T}{T}\right)^{2}-\frac{\tau_{\mathrm{q}}}{\lambda_{\mathrm{q}} T^{2}}\left(\delta \mathbf{J}_{\mathrm{q}}\right)^{2}+\frac{4 \tau_{\mathrm{q}}}{\lambda_{\mathrm{q}} T^{3}} \mathbf{J}_{\mathrm{q}} \cdot \delta \mathbf{J}_{\mathrm{q}} \delta T-\frac{3 \tau_{\mathrm{q}}}{\lambda_{\mathrm{q}} T^{4}}\left(\mathbf{J}_{\mathrm{q}}\right)^{2}(\delta T)^{2} .
$$

Notice that when $\tau_{\mathrm{q}}=0$, the classical value (10), is obtained. The second differential of the classical entropy is always negative definite, and hence can be chosen as a Lyapunov's functional. This property is, in general, not always fulfilled for the generalized entropy.

The solution of the model is obtained by means of the spectral method. Thus, the zonally averaged temperature in degrees Celsius, insolation and co-albedo are assumed to follow an expansion in Legendre polynomials (North 1975b):

$$
\begin{aligned}
& \mathscr{F}(t, x)=\mathscr{F}_{0}(t)+\mathscr{F}_{2}(t) P_{2}(x), \\
& S(x)=1+S_{2} P_{2}(x), \\
& a(x)=a_{0}+a_{2} P_{2}(x),
\end{aligned}
$$

where $P_{2}(x)$ is the second-order Legendre polynomial, and the first order drops out by taking symmetric hemispheres into account. Thus, $\Phi_{0}(t)$ is the planetary globally averaged temperature and $\mathscr{I}_{2}(t)$ is 1.5 times the temperature difference between pole and equator, $S_{2}=-0.477$ due to the dependence of $P_{2}(x)$ on $x$. Equation (20c) is used for latitudes equatorwards of the ice-sheet edge $\left(a_{0}=0.697, a_{2}=-0.0779\right)$. At higher latitudes a constant co-albedo (equal to the ice value $\approx 0.38$ ) is assumed. Using $(20 \mathrm{a})$ and $(5)$ with the expression of the gradient operator (3a) and ( $3 b$ ), the heat flux takes the form:

$$
\mathbf{J}_{\mathrm{q}}(t, x)=-\lambda_{\mathrm{q}} \mathscr{q}_{\mathrm{q}}(t) x\left(1-x^{2}\right)^{1 / 2} .
$$

$\mathscr{F}_{\mathrm{q}}(t)$ has units of degrees Celsius, and is equal to $\mathscr{F}_{2}(t)$ at the steady state (Pujol and Llebot 1999) with $\lambda_{\mathrm{q}}=0.591 \mathrm{~W} \mathrm{~m}^{-2} \mathrm{~K}^{-1}$. For an ice-variable earth, $\mathscr{I}_{0}$ and $\mathscr{I}_{2}$ are linked through the ice-sheet condition:

$$
\mathscr{F}_{0}+\mathscr{F}_{2} P_{2}\left(x_{\mathrm{s}}\right)=-10^{\circ} \mathrm{C},
$$

where $x_{\mathrm{s}}$ is the sine of the latitude of the ice-sheet edge. 


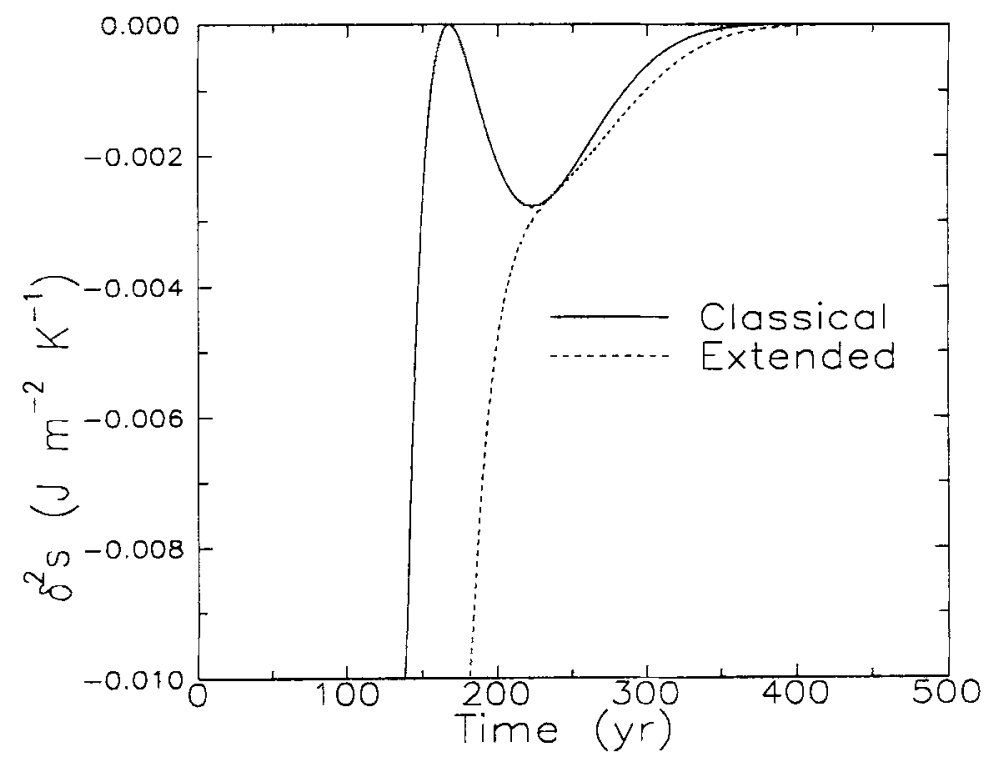

Figure 1. Time evolution of the second differential of entropy, $\delta^{2} s$, for an ice-free earth. The case where the response time $\tau_{\mathrm{q}} \approx 40$ years. A stable steady state is only obtained for any response time following the extended expression (monotonically growing). The classical expression leads to unstable steady states for response times higher than 21 years. Initial conditions: $\Phi_{0}=14.46^{\circ} \mathrm{C}, \Phi_{2}=\Phi_{\mathrm{q}}=-30.0^{\circ} \mathrm{C}$. For definitions and other values see section 2 .

\section{RESULTS}

Time evolutions for $\delta^{2} s$ and $\delta^{2} s^{*}$ have been obtained from (1) and (5) using the development described in (20) to (22). The initial $2 \times 2$ matrix $\left(\delta T, \delta \mathbf{J}_{\mathrm{q}}\right.$ ) observed in (19) becomes a $3 \times 3$ matrix when the latitudinal integration is carried out and equations (20a) and $(21)$ are taken into account $\left(\delta \mathscr{F}_{0}, \delta \mathscr{F}_{2}, \delta \Phi_{\mathrm{q}}\right)$. The time-dependent evolution for both an ice-free and ice-variable earth follows a standard Runge-Kutta method of numerical integration. The time step is taken as $\sim 2$ months in order to obtain enough accuracy within the long time-scale analysed $\left(C \approx 10^{10} \mathrm{~J} \mathrm{~m}^{-2} \mathrm{~K}^{-1}\right.$, Bhattacharya et al. 1982).

\section{(a) Ice-free earth}

In this case the global planetary albedo has been chosen as 0.303 (Nicolis and Nicolis 1980). The results obtained for a response time like that assumed for the mixed ocean layer $\left(\tau_{\mathrm{q}} \approx 1 \mathrm{yr}\right.$ ) show a monotonically growing second differential of the entropy for both expressions. Then, the steady state becomes stable. On the other hand, an increase in the response time, implies a non-monotonically growing $\delta^{2} s$, as we can see in Fig. 1 . In this case, $\tau_{\mathrm{q}} \approx 40 \mathrm{yr}$. As Pujol and Llebot (1999) show, high values of delay time produce a non-monotonic behaviour of $\mathscr{F}_{2}$, with several extremal values different to the stationary one. For an ice-free earth $\mathscr{F}_{0}$ is independent of the value chosen for the response time. Hence, $\mathscr{F}_{0}$ has been maintained fixed through the time evolution. Therefore, the zero values for the classical expression of $\delta^{2} s$ in Fig. 1 correspond to extremal points in $\mathscr{I}_{2}$. The steady state, then, would be unstable following the classical criteria of the second differential of the entropy. The additional terms in (19) for the generalized entropy compensate these oscillations for any value of the response time. In fact, a climate without ice-albedo feedback always reaches the steady state whatever response time is chosen. Hence, one expects the stability result for any response time used. The lower value of the 


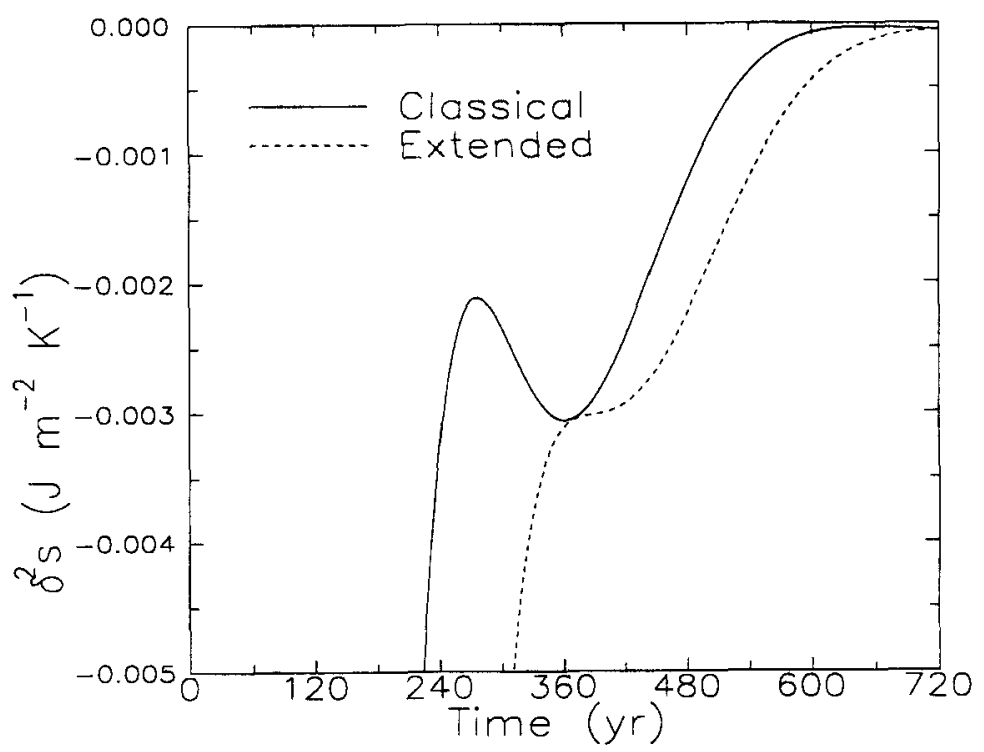

Figure 2. Time evolution of the second differential of entropy, $\delta^{2} s$, for an ice-variable earth, for the case where the response time $\tau_{\mathrm{q}} \approx 82$ years. The extended expression leads to a stable steady state, whereas in the range 42 years $<\tau_{\mathrm{q}}<81$ years unstable states are obtained. The classical solution gives unstable states up to 41 years for $\tau_{\mathrm{q}}$. Initial conditions: $\mathscr{F}_{0}=14.9^{\circ} \mathrm{C}, \mathscr{F}_{2}=\mathscr{q}_{\mathrm{q}}=-30.0^{\circ} \mathrm{C}$. For definitions and other values see section 2.

response time where stable steady states are obtained by following the classical criteria is $\sim 21$ years. Thus, the results show the domain of applicability of the extended expression is much larger, and that corresponds better with the stability of the steady states than the classical expression. This fact confirms the choice of the generalized entropy in nonequilibrium conditions. Different initial conditions in the system do not imply changes in the behaviour of the time evolution for the second differential of the entropy, although an increase in the initial heat flux can generate a non-negative definite $\delta^{2} s^{*}$. For this reason, we have only considered values of the initial heat flux close to the steady state, in agreement with the first-order approach shown in (5).

\section{(b) Ice-albedo feedback}

In this case we have taken the ice-albedo feedback into account by assuming possible variations in ice-caps. The albedo introduced in the model is in the form of a step function, where for latitudes higher than the ice-sheet, the albedo is constant and equal to the value of the ice albedo. As in $(a)$, for a response time $\approx 1$ year, results for both classical and generalized entropy are the same, and lead to a stable steady state. The initial conditions from which the present state is reached are taken as: $\mathscr{\Phi}_{0}(t=0)$ equal to the steady state value $\left(14.9^{\circ} \mathrm{C}\right)$; and $\mathscr{F}_{2}(t=0)$ a slightly less homogeneous $\left(-30^{\circ} \mathrm{C}\right)$. The results show a little variation with changes in $\Phi_{2}(t=0)$ for a fixed $\Phi_{0}(t=0)$. For delay times longer than 45 years, the second differential of the classical entropy grows non-monotonically. This would indicate instability at stationary conditions. The extended expression, however, splits the whole space of $\tau_{\mathrm{q}}$ values into different stable regions. Thus, for $\tau_{\mathrm{q}}<42$ years the criteria of $\delta^{2} s^{*}$ gives stable steady states. Furthermore, a narrow band of delay times, 81 years $<\tau_{\mathrm{q}}<84$ years also indicates stability. This case is shown in Fig. 2, where the classical expression does not reach the zero value as in $(a)$ due to the lag between both $\mathscr{F}_{0}$ and $\mathscr{F}_{2}$ non-monotonic evolutions. In this case, the nonlinearity obtained through 
the condition of variable ice-sheets (22) can produce self-sustained oscillations for high response times ( $\sim 370$ years; Pujol and Llebot 1999). Then, in the generalized case, higher values of response time do not necessarily imply higher likelihood of obtaining the unstable solution. For example, a stable state is found when $\tau_{\mathrm{q}}=82$ years whereas an unstable state is obtained for $\tau_{\mathrm{q}}=50$ years. It indicates that the current climate does not present a continuity in the possible states reached; i.e. not all the distributions of meridional heat transport (7) correspond to stable climates. However, if the initial state corresponds to a different globally averaged temperature, the solutions vary. Thus, for an initial difference of pole-equator temperatures like that for the steady state but with a colder mean planetary temperature $\left(\mathscr{F}_{0}(t=0)=14.2^{\circ} \mathrm{C}\right)$ the range of stable steady states for both classical and extended expression is up to 27 years. In this case, no stable solution for the extended expression beyond 27 years has been found. A change in solar constant also modifies the solutions found. Thus, in the ice-age state $\left(Q=335 \mathrm{~W} \mathrm{~m}^{-2}, \mathscr{I}_{0}=8.9^{\circ} \mathrm{C}, \mathscr{F}_{2}=-32.4^{\circ} \mathrm{C}\right)$ the initial conditions have been taken as $\mathscr{\Phi}_{0}(t=0)=8.9{ }^{\circ} \mathrm{C}$ and $\Phi_{2}(t=0)=-34{ }^{\circ} \mathrm{C}$ in order to facilitate the comparison with the previous results. In this case, beyond a response time of 25 years both classical and extended expressions give unstable solutions. A colder climate, therefore, would be more sensitive to internal changes; i.e. the dynamical states ( $\tau_{\mathrm{q}}$ values) which lead to stable solutions would diminish in comparison with the current case.

For high values of response time, the development to first order of Fourier's law is not a good approach and, consequently, it would be necessary to modify the expression for the generalized entropy. Nevertheless, the self-sustained oscillations which result beyond a particular value of response time, imply the irrelevance of the second differential of the entropy as a measure for the climatic stability in such region.

Furthermore, we have not evaluated the contribution of radiative fluxes to the generalized entropy. These terms would play an important role, since variations in the ice-caps imply variations in both short-and long-wave radiation. However, for a horizontal vertically averaged model the introduction of this contribution is extremely difficult (Pujol and Llebot 1998).

\section{EXTREMAL RATE OF ENTROPY PRODUCTION}

Following the thermodynamic analysis of the climatic system, it is interesting to obtain the extremal rates of entropy production. Prigogine (1947) shows that particular systems (but not the climate) tend to a state of minimum rate of entropy production. If the climatic system satisfied a similar condition, we would avoid the assumption of some parametrizations and the uncertainties that they imply. Therefore, many authors have tried to estimate the climatic rate of entropy production. Paltridge $(1975,1978)$ developed a box model, which uses the constraint of maximum dissipation rate for the material contribution as a fundamental hypothesis. The results produce reasonable distributions of temperature, cloud-cover and meridional heat fluxes (see also Grassl 1981). A detailed analysis of Paltridge's model is carried out by O'Brien and Stephens (1995) in order to observe its predictive relevance. On the other hand, global entropy production, which only contains the radiative exchange along the planetary boundaries, is found to be very insensitive to different dynamical states. Thus, Lesins (1990) found that the present value of global entropy production is only $1 \%$ less than that for an hypothetical latitudinally isothermal earth. Recently, O'Brien (1997) found an insignificant difference in global entropy production between two opposite dynamical cases (zero transport and uniform earth) for the whole range of possible albedos. 
Here, we find the extremal value of global entropy production as a function of the albedo for a 1D EBM. Stephens and O'Brien (1993) have already obtained it, but by using a zero-dimension climatic model. The maximum case corresponds to that with uniform temperature, so the comparisons with current values are in accordance with Lesins (1990) and O'Brien (1997). The material entropy production, therefore, appears to be dynamically interesting with the maximum values also calculated as functions of the albedo. Wyant $e t$ al. (1988) also obtain the maximum rate of entropy production for the present situation, but by assuming a diffusive approach for the heat flux. Here, the extremal values are calculated using the variational principle (e.g. Goldstein 1950). This idea has already been developed by Nicolis and Nicolis (1980) who obtain the heat flux which corresponds to an extremal value of the rate of entropy production. These authors, however, consider the entropy production without any radiative contribution. Furthermore, a comparison with ice-age conditions (for both extremal and current values) has been carried out, following $\mathrm{Li}$ and Chýlek (1994) who describe the entropy production in those scenarios.

At the steady state, classical and extended expressions for the rate of entropy production become the same. Thus, equation (15) yields:

$$
\mathscr{P}=\nabla \cdot \mathbf{H}-\frac{\nabla \cdot \mathbf{R}}{T}+\mathbf{J}_{\mathrm{q}} \cdot \nabla \frac{1}{T}
$$

The radiation flux of entropy $\mathbf{H}$ is not linearly related to the radiation flux of energy R (e.g. Landsberg and Tonge 1979; Stephens and O'Brien 1993). Thus, the energy flux density $R$ and the entropy flux density $H$ for a surface of temperature $T$ with emissivity $\omega$ follow:

$$
\begin{aligned}
& R=\sigma T^{4} \omega, \\
& H=\frac{4}{3} \sigma T^{3} \chi(\omega),
\end{aligned}
$$

where $\sigma$ is Stefan's constant and $0 \leqslant \chi(\omega) \leqslant 1$ with $\chi(1)=1$ and $\chi(0)=0$.

Stephens and O'Brien (1993) show that for emitting surfaces the approximation $\chi(\omega) \sim \omega$ can be accepted, i.e. for incoming short-wave and outgoing long-wave radiation. In this case an expression linking the entropy with the energy flux of radiation can be assumed (Li and Chýlek 1994):

$$
\nabla \cdot \mathbf{H}=\frac{4}{3} \frac{\nabla \cdot \mathbf{R}}{T_{\mathrm{eff}}}
$$

where $T_{\text {eff }}$ is $T$ for long-wave outgoing radiation. Hence, from ( $\left.3 a\right)$ and ( $3 b$ ):

$$
\begin{aligned}
\nabla \cdot \mathbf{H}_{1-} & =\frac{4}{3} \frac{A+B T-B 273}{T}, \\
\nabla \cdot \mathbf{H}_{\text {s- }} & =-\frac{4}{3} \frac{Q S}{T_{\text {sun }}} .
\end{aligned}
$$

where $T_{\text {sun }}$ is $T(\approx 5777 \mathrm{~K})$ for short-wave incoming radiation. However, for low values of $\omega$-let us say outgoing short-wave radiation, (25) becomes incorrect, and the following function for $\chi(\omega)$ can be used:

$$
\chi(\omega)=\omega\{u \log (\omega)+v\},
$$

with $u \approx-0.277$ and $v \approx 0.965$ (Stephens and O'Brien 1993, Goody and Abdou 1996). 
In meridional 1D models, (27) is zonally averaged, which yields:

$$
\psi(\omega)=\omega\{u \log (2 \omega)+v-u\}
$$

where now only $\omega$ keeps its latitudinal dependence (see O'Brien 1997).

Due to the outgoing short-wave radiation it follows that:

$$
Q S(1-a)=\sigma T_{\mathrm{sun}}^{4} \frac{\Omega}{4} S(1-a),
$$

where the factor $1-a$ is the albedo, and $\Omega$ the solid angle subtended by the Sun. From (24a) $\omega$ becomes:

$$
\omega=\frac{\Omega}{4} S(1-a)
$$

whereby from (24b) and (29) the outgoing entropy flux of radiation $\nabla \cdot \mathbf{H}_{\mathrm{s}+}$ yields:

$$
\nabla \cdot \mathbf{H}_{\mathrm{s}+}=\frac{4}{3} \frac{Q S(1-a)}{T_{\text {sun }}}\left[u \log \left\{2 \frac{\Omega}{4} S(1-a)\right\}+v-u\right] .
$$

From (26a), (26b) and the above, (23) can be expressed as:

$$
\mathscr{P}=\frac{1}{3} \frac{A+B T-273 B}{T}-\frac{4}{3} \frac{Q S}{T_{\text {sun }}}+\frac{Q a S}{T}+\nabla \cdot \mathbf{H}_{\mathrm{s}+}+\mathbf{J}_{\mathrm{q}} \cdot \nabla \frac{1}{T},
$$

where the term involving the factor $1 / 3$ represents the long-wave contribution of the two first terms on the r.h.s. in (23). The term with the factor $4 / 3$ is the incoming short-wave entropy flux of radiation, whereas the third term on the r.h.s. corresponds to the short-wave part of the second term in (23).

At the steady state, $\mathscr{P}$ is a function of only $\mathbf{J}_{\mathfrak{q}}, \partial \mathbf{J}_{\mathrm{q}} / \partial x$ and $x$. From (1) the temperature as a function of $\mathbf{J}_{\mathrm{q}}$ and $x$ takes the value:

$$
T=\left\{g(x)-\nabla \cdot \mathbf{J}_{\mathrm{q}}\right\} \frac{1}{B},
$$

where the function $g(x)$ is defined as:

$$
g(x)=Q a\left(x_{\mathrm{s}}, x\right) S(x)-A+273 B .
$$

Now, the heat flux $\mathbf{J}_{\mathbf{q}}$, which gives an extremum of the global rate of entropy production, can be obtained by using variational techniques. The solution gives:

$$
\nabla \cdot \mathbf{J}_{\mathrm{q}}=g(x)+k
$$

where the value for $k$ is found by taking into account the condition of zero heat flux at the boundaries of the system:

$$
k=-\int_{0}^{1} g(x) \mathrm{d} x .
$$

For the material entropy production, the extremal heat flux follows Nicolis and Nicolis (1980):

$$
\nabla \cdot \mathbf{J}_{\mathrm{q}}=g(x)-\frac{\int_{0}^{1} g(x) \mathrm{d} x}{\int_{0}^{1} g(x)^{1 / 2} \mathrm{~d} x} g(x)^{1 / 2} .
$$




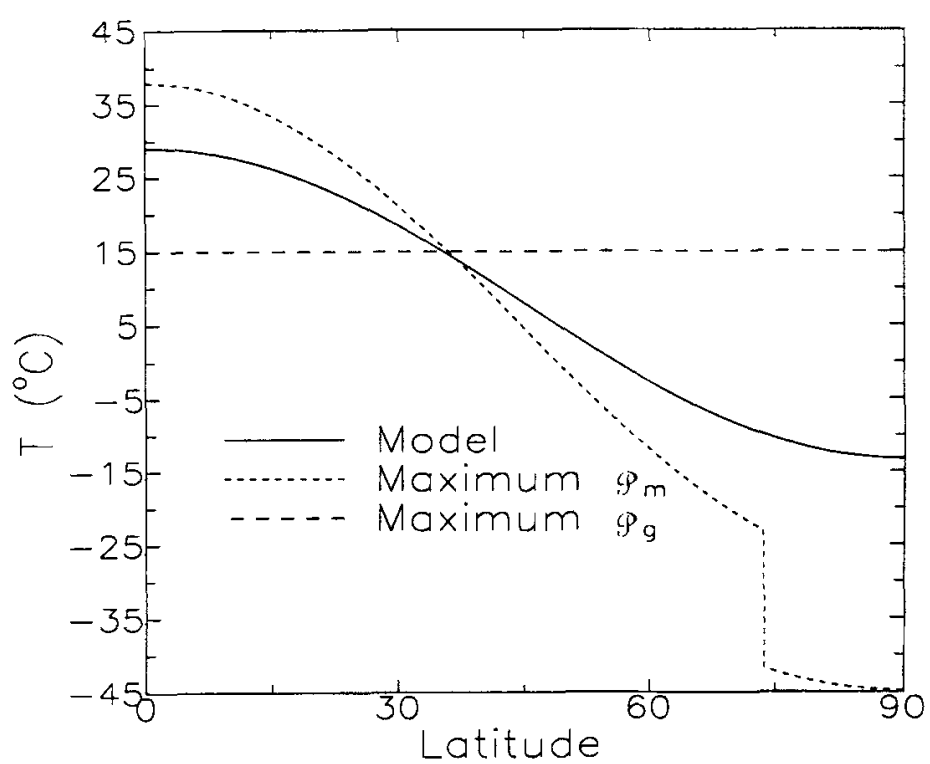

Figure 3. Current latitudinal distribution of temperature, $T$, compared with those obtained for both material, $\mathscr{P}_{\mathrm{m}}$, and global, $\mathscr{P}_{\mathrm{g}}$, maximum entropy production. The step at the ice-sheet edge for the extremal cases is due to the assumption of a step function for the albedo.

In Fig. 3 the latitudinal distribution of temperature for the maximum rates of both material entropy, $\mathscr{P}_{\mathrm{m}}$, and global entropy, $\mathscr{P}_{\mathrm{g}}$, production are shown. The step at the icesheet latitude in the material entropy case is because the extremal distributions are functions only of the radiative parameters. Thus the abrupt change in the albedo is observed in latitudinal distributions of temperature, as well as in both extremal entropy production and heat flux.

The maximum value for the global entropy production corresponds to the isothermal state, whereas for the material entropy production the distribution becomes less homogeneous than the present. This fact agrees with the decrease in conductivity found by Wyant et al. (1988) at the maximum state of material entropy production if the diffusivity approach is maintained.

In Fig. 4, the maximum rate of global entropy production is shown for current and ice-age conditions. Present global entropy production obtained by using (32) and Fourier's law (4) is shown; a value $\approx 1.133 \mathrm{~W} \mathrm{~m}^{-2} \mathrm{~K}^{-1}$ is found. Essex (1984) obtains $1.177 \mathrm{~W} \mathrm{~m}^{-2} \mathrm{~K}^{-1}$, whereas Stephens and O'Brien (1993) find $1.25 \mathrm{~W} \mathrm{~m}^{-2} \mathrm{~K}^{-1}$ from satellite measurements. In addition, we have plotted the value for the entropy production in the last ice-age using a value for the solar constant of $Q=335 \mathrm{~W} \mathrm{~m}^{-2}$ when the model gives a planetary temperature of $\Phi_{0}=8.9^{\circ} \mathrm{C}$, a latitudinal distribution of temperatures $\Phi_{2}=-32.4{ }^{\circ} \mathrm{C}$ and an ice-sheet edge $x_{\mathrm{s}}=0.84$. In these conditions, the global entropy production is $1.115 \mathrm{~W} \mathrm{~m}^{-2} \mathrm{~K}^{-1}$. Thus, global entropy production decreases when globally averaged temperature decreases, in agreement with $\mathrm{Li}$ and Chýlek (1994). The respective maximum values are less than $0.3 \%$ higher, being $1.135 \mathrm{~W} \mathrm{~m} \mathrm{~W}^{-2} \mathrm{~K}^{-1}$ and $1.118 \mathrm{~W} \mathrm{~m}^{-2} \mathrm{~K}^{-1}$ for the present and ice-age conditions respectively. This result also agrees with Lesins (1990) and O'Brien (1997) if one assumes the isothermal distribution in those studies as the state of maximum entropy production. For all possible values of the ice-sheet edge, the difference between both extremal cases remains practically unchanged $\left(\approx 0.017 \mathrm{~W} \mathrm{~m} \mathrm{~m}^{-1}\right)$. 


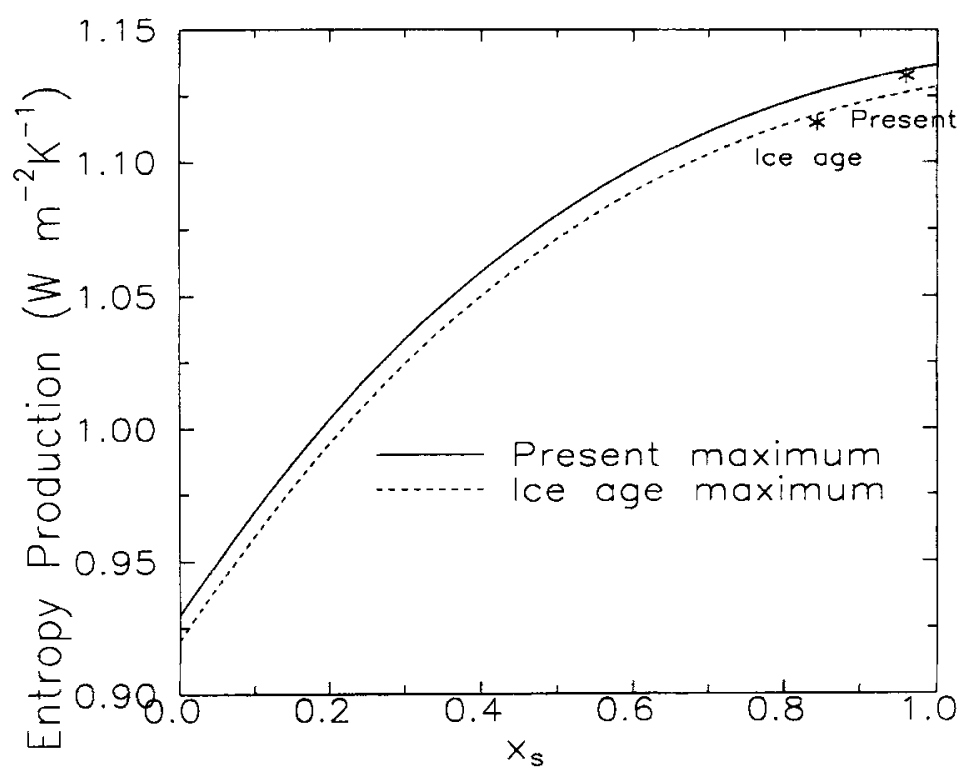

Figure 4. Maximum global entropy production for present and ice-age conditions as a function of the planetary albedo; $x_{\mathrm{s}}$ is the sine of the latitude of the ice edge. The specific values in those scenarios are plotted $\left(^{*}\right)$. See text for further details.

The 1D model analysed, produces two more possible steady states for current conditions. A stable fully ice-covered earth $\left(\mathscr{F}_{0}=-54^{\circ} \mathrm{C}, \Phi_{2}=-12{ }^{\circ} \mathrm{C}\right)$ and an intermediate unstable state $\left(\mathscr{F}_{0}=-20^{\circ} \mathrm{C}, \mathscr{F}_{2}=-35^{\circ} \mathrm{C}\right.$, ice-sheet $\left.=21.5^{\circ}\right)$. The global entropy production for the unstable state differs by $\sim 0.4 \%$ of the maximum value, whereas for the fully ice-covered earth it differs only by $0.1 \%$ of the maximum value.

In Fig. 5, the present and ice-age maximum values for material entropy production are shown as functions of the albedo. Contrary to the global case, the differences between both expressions vary as functions of planetary albedo. For present conditions $\left(Q=340 \mathrm{~W} \mathrm{~m}^{-2}\right)$ the maximum value is $\approx 0.0087 \mathrm{~W} \mathrm{~m}^{-2} \mathrm{~K}^{-1}$, and for the last ice-age $\approx 0.0126 \mathrm{~W} \mathrm{~m}^{-2} \mathrm{~K}^{-1}$; these are below the upper bounds proposed by Essex $(1984)$ of $\approx 0.0112 \mathrm{~W} \mathrm{~m}^{-2} \mathrm{~K}^{-1}$ and $\approx 0.0144 \mathrm{~W} \mathrm{~m}^{-2} \mathrm{~K}^{-1}$ for present conditions and the last ice-age respectively. In regard to the climatic values given for the model, the material entropy production at the ice-age becomes larger than the present value, because the pole-equator temperature differences are greater in the ice-age $\left(0.0099 \mathrm{~W} \mathrm{~m}^{-2} \mathrm{~K}^{-1}\right.$ versus $\left.0.0070 \mathrm{~W} \mathrm{~m}^{-2} \mathrm{~K}^{-1}\right)$. In this case, differences with extremal values are higher than $20 \%$. This is evident since the system analysed is not very close to the maximum rate of material entropy production. Nevertheless, the difference increases up to $46 \%$ for the unstable solution.

It can be seen that the material entropy production is substantially lower than the global entropy. In fact, the global entropy production is related to the exchange of radiation flux of entropy with the surroundings. Hence, both short- and long-wave radiation contribute to the global entropy production. However, both contributions clearly show an imbalance; the short-wave incoming radiation presents a high efficiency ( $T$ emission $\sim 5777 \mathrm{~K}$ ) with a low rate of entropy production. The reflected short-wave radiation loses an important part of this efficiency (Li et al. 1994; O'Brien 1997) and its rate of entropy production becomes higher, even, than the incoming radiation. The low efficiency of the outgoing long-wave radiation causes, therefore, the high rate of entropy production. On the other hand, the material entropy production in the low-dimensional model analysed is only a function of 


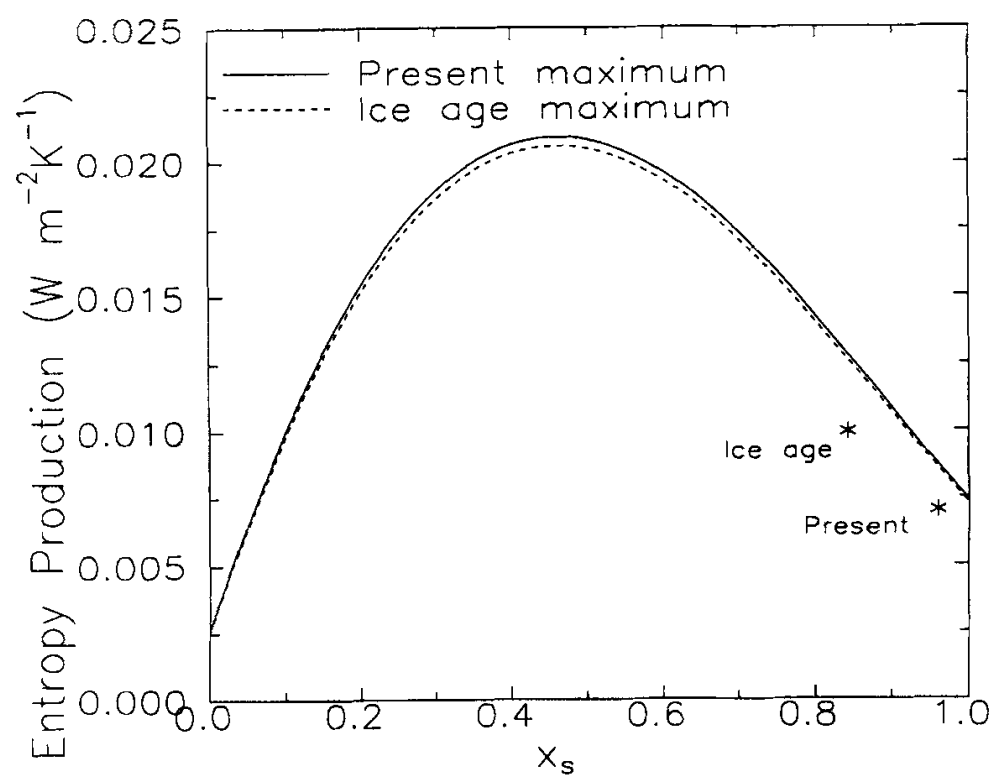

Figure 5. Maximum material entropy production for present and ice-age conditions as a function of the planetary albedo; $x_{5}$ is the sine of the latitude of the ice edge. The specific values in those scenarios are plotted $\left(^{*}\right)($ see text). In this case the difference with regard to maximum values increases up to $20 \%$.

meridional heat fluxes, which are the residual of the latitudinal (low) imbalance between outgoing and incoming radiation energy.

The globally averaged value of the global entropy production is, in accordance with observations and estimations, obtained by applying different climatic models. However, the globally averaged material entropy production is considerably lower than that expected (e.g. Peixoto et al. 1991). This is because the low-dimensional 1D model analysed does not take the latent- and sensible-heat flux into account.

\section{CONCLUding REMARKS}

In this paper, the second differential of the entropy is applied to a thermodynamic 1D model to study the stability of the steady states by taking the heat fluxes as independent variables. The evolution equation of the fluxes has been assumed to follow a MaxwellCattaneo type equation, which represents an extension of Fourier's law beyond stationary conditions. The introduction of more general evolution equations, however, can be qualitatively reduced to a Maxwell-Cattaneo form, which uses a delay time for heat fluxes (Jou et al. 1996). For an ice-free earth, any value of this response time leads to the same steady state (Pujol and Llebot 1999). When variable ice-caps are taken into account, however, self-sustained oscillations can be obtained by assuming a response time higher than a given critical value. The stability of the steady states for different response times is classified through the second differential of the entropy. Although the numerical values of the steady states are the same, their stability differs as a function of the response time used. In fact, this response time is a measure of the climatic dynamics. Thus, the climatic stability as a function of different dynamical regimes is obtained with a very simple analysis.

Moreover, an extended expression for the climatic entropy (a function of temperature and fluxes) has been developed, following the principles assumed in the extended irreversible thermodynamics theory (EIT). The classical expression of the second differential 
of the entropy (a function of temperature only) is chosen as a Lyapunov's functional because of its negative definite sign. The extended expression can present problems, with its sign depending on the value used for the heat flux (see Jou et al. 1996). Nevertheless, for realistic initial conditions the extended expression is also negative definite.

By using a response time of $\sim 1$ year related to the mixed ocean layer, both expressions grow monotonically implying the stability of the steady state, with and without ice-albedo feedback within the model. For an ice-free earth, higher values of the response time (up to 21 years) lead to anomalous behaviour of the classical expression. Conversely the extended expression leads to stable states for any response time.

By including the ice-albedo feedback, the second differential of the classical entropy gives unstable stationary states when the response time is higher than 45 years. The extended expression, however, shows some regions with stable states for high values of response time. Thus, for $\tau_{\mathrm{q}}$ between 42 and 81 years the extended analysis produces unstable steady states. Nevertheless, in a narrow band of 81 years $<\tau_{\mathrm{q}}<84$ years stability of the state is reached. This result indicates that no distribution of heat transport (related to $\tau_{q}$ ) leads to stable climates when the ice-albedo feedback is included.

Previous values have been obtained by assuming an initial state with a planetary temperature equal to the steady state temperature. Changes related to the pole-equator differences in the initial conditions do not substantially modify these results. However, important variations are observed by changing the initial mean planetary temperature. An evolution, for instance, from a colder climate $\left(\Phi_{0}(t=0)=14.2{ }^{\circ} \mathrm{C}\right)$ produces unstable steady states for a response time higher than 27 years for both expressions. Furthermore, the region of stable states also changes for different climatic conditions. For the ice-age, the calculations give unstable states beyond 25 years of response time for both classical and extended expressions on applying a similar evaluation as for the present case. Therefore, the number of stable states (range of $\tau_{\mathrm{q}}$ values) in a colder climate is reduced. In general, the extended expression can be applied over a larger range than the classical one. This range could be even more extended with the assumption of radiative fluxes in the generalized entropy. This contribution, however, is difficult to evaluate in a 1D meridional model.

Following the thermodynamic analysis of this climatic system, the extremal rate of entropy production is obtained through the variational principle (Nicolis and Nicolis 1980). The maximum rate of global entropy production, which is a function only of the radiation flux of entropy, is obtained. This case is found to correspond to an isothermal state whereby slight variations from current values are expected (Lesins 1990; O'Brien 1997). Our calculation gives $\sim 0.3 \%$ difference between maximum and current values of global entropy production. Hence, the low sensitivity of this expression to the climatic dynamics can be seen. A reduction in solar constant $\left(Q=335 \mathrm{~W} \mathrm{~m}^{-2}\right.$, last ice-age) produces a uniform decrease of the maximum rate of global entropy production corresponding to the possible values for the ice-sheet edge. In addition, the maximum value for the extremal global entropy production is obtained for an ice-free earth in agreement with Stephens and O'Brien (1993). The value for the ice-age condition is also lower than that for the present day, due to the decrease in the mean planetary temperature.

Paltridge $(1975,1978)$ hypothesises a maximum-dissipation principle related to the material contribution, once the possible irrelevance of global entropy production for defining the dynamics is observed. Thus, plausible values of temperature, cloud cover and meridional heat fluxes are found in a 1D box model through finding the extremum of material entropy production. Nevertheless, until now there has been no theoretical justification of this hypothesis. Here, the values of the maximum rate of material entropy production are found as a function of the ice-sheet edge. In this case, the latitudinal distribution of temperatures is less homogeneous than present conditions, following Wyant 
et al. (1988) who obtained a decrease in the conductivity through maximising the material entropy production. These authors, however, consider a diffusive approach for the heat flux. As in the case of global entropy production, the maximum rate of material entropy production is lower for the ice-age. The maximum in the extremal distribution is found to be at middle values for the ice-sheet edge, where the climatic model is expected to give the maximum pole-equator differences of temperature. Moreover, the material entropy production at the ice-age is greater than the present due to the higher difference between pole-equator temperatures in the former. The maximum values for present and ice-age conditions are lower than the upper bound proposed by Essex (1984). Current values, however, differ by at least $20 \%$ with respect to the maximum possible levels. In the light of these results, the climate does not seem to reach a state of maximum material entropy production. However, by analysing the three possible steady states for current conditions, the present state is much closer to the maximum value than the unstable one, but not in reference to the ice-covered earth. However, this last case produces a substantially lower material entropy production than the current state. Thus, it is still unclear how the climatic system evolves, leaving open the possibility of an extremal entropy behaviour.

\section{ACKNOWLEDGEMENT}

This work has been partially supported by the Ministerio de Educación y Cultura of the Spanish government under contracts CL195-1867 and PB96-0451.

Bhattacharya, K., Ghil, M. and Vulis, I. L.

Cahalan, R. F. and North, G. R.

Callies, U. and Herbert, F.

Criado-Sancho, M. and Llebot, J. E. 1993

Dedeurwaerdere, T., Casas-Vázquez, J., Jou, D. and Lebon, $\mathrm{G}$.

Essex, C.

Ghil, M.

Goldstein, $\mathbf{H}$.

Goody, R. and Abdou, W.

Grassl, H.

Hasselmann, K.

Jou, D., Casas-Vázquez, J. and Lebon, G.

Landsberg, P. T. and Tonge, G.

Lesins, G. B.

Li, J. and Chýlek, P.

Li, J., Chýlek, P. and Lesins, G. B.

\section{REFERENCES}

1982

1979

1988

1996

1984

1976

1950

1996

1981

1979

1996

1979

1990

1994

1994
Internal variability of an energy-balance model with delayed albedo effects. J. Atmos. Sci., 39, 1747-1773

A stability theorem for energy-balance climate models. J. Atmos. Sci., 36, 1178-1188

Radiative processes and non-equilibrium thermodynamics. $J$. Appl. Math. Phys., 39, 242-266

Behavior of entropy in hyperbolic heat conduction. Phys. Rev. E., 47, $4104-4107$

Foundations and applications of a mesoscopic thermodynamic theory of fast phenomena. Phys. Rev. E., 53, 498-506

Radiation and irreversible thermodynamics of climate. J. Atmos. Sci., 41, 1985-1991

Climate stability for a Sellers-type model. J. Atmos. Sci., 33, 2-20

Classical mechanics. Addison-Wesley, Massachusetts, USA

Reversible and irreversible sources of radiation entropy. $Q . J . R$. Meteorol. Soc., 122, 483-494

The climate at maximum entropy production by meridional atmospheric and oceanic heat fluxes. Q.J. R. Meteorol. Soc., 107, 153-166

'Some comments on the design of model response experiments for multi-time-scale systems'. Pp. 1037-1049 in Report of the JOC study conference on climate models: Performance, intercomparison and sensitivity studies, GARP Publ, SER., No 22, WMO/ICSU, Geneva

Extended irreversible thermodynamics. Springer-Verlag, Berlin, Germany

Thermodynamics of the conversion of diluted radiation. J. Phys. A., 12, 551-562

On the relationship between radiative entropy and temperature distributions. J. Atmos. Sci., 47, 795-803

Entropy in climate models. Part II: Horizontal structure of atmospheric entropy production. J. Atmos. Sci., 51, 1702-1708

Entropy in climate models. Part I: Vertical structure of atmospheric entropy production. J. Atmos. Sci., 51, 1691-1701 
Nicolis, G. and Nicolis, C.

North, G. R.

O’Brien, D. M.

O'Brien, D. M. and Stephens, G. L. 1995

Paltridge, G. W.

Peixoto, J. P., Oort, A. H., de Almeida, M. and Tomé, A. Prigogine, I.

Pujol, T. and Llebot, J. E.

Stephens, G. L. and O'Brien, D. M.

Su, C. H. and Hsieh, D. Y.

Wyant, P. H., Mongroo, A. and Hameed, S.
1980 On the entropy balance of the earth-atmosphere system. $Q . J . R$. Meteorol. Soc., 106, 691-706

1975a Analytical solution to a simple climate model with diffusive heat transfer. J. Atmos. Sci., 32, 1301-1307

1975b Theory of energy-balance models. J. Atmos. Sci., 32, 2033-2043

1997 A yardstick for global entropy-flux. Q. J. R. Meteorol. Soc., 123, $243-260$

Entropy and climate. II: Simple models. Q. J. R. Meteorol. Soc., 121, 1773-1796

1975 Global dynamics and climate-a system of minimum entropy exchange. Q. J. R. Meteorol. Soc., 101, 475-484

1978 The steady-state format of global climate. Q. J. R. Meteorol. Soc., 104, 927-945

1991 Entropy budget of the atmosphere. J. Geophys. Res., 96, 1098110988

1947 Etude thermodynamique des phénomènes irreversibles. Desoer, Liège, Belgium

1998 Entropy production in thermodynamic climatic models. J. NonEquilibrium Thermody, 23, 62-86

1999 Periodic solutions in low-dimensional climatic models. J. Climate, in press

1993 Entropy and climate. I: ERBE observations of the entropy production of the earth. Q.J.R. Meteorol. Soc., 119, 121-152

1976 Stability of the Budyko climate model. J. Atmos. Sci., 33, 22732275

1988 Determination of the heat-transport coefficient in energy-balance climate models by extremization of entropy production. $J$. Atmos. Sci. , 45, 189-193 